\title{
In Vitro and in Vivo Antitrypanosomal Activitiy of Two Microbial Metabolites, KS-505a and Alazopeptin
}

\author{
Aki Ishiyama, Kazuhiko Otoguro, Miyuki Namatame, Aki Nishihara, \\ Toshiaki Furusawa, Rokuro Masuma, Kazuro Shiomi, Yoko Takahashi, \\ Michio Ichimura, Haruki Yamada, Satoshi Ōmura
}

Received: July 22, 2008 / Accepted: September 15, 2008

(C) Japan Antibiotics Research Association

\begin{abstract}
Our on-going screening program to discover new antitrypanosomal antibiotics has been evaluating compounds isolated from soil microorganisms as well as investigating the antibiotic libraries of the Kitasato Institute for Life Sciences and BioFrontier Laboratories of Kyowa Hakko Kogyo Co., Ltd. We have now discovered two compounds, KS-505a and alazopeptin, which exhibit moderate antitrypanosomal characteristics. We report here the in vitro and in vivo antitrypanosomal activities and cytotoxicities of KS-505a and alazopeptin, compared with some commonly-used antitrypanosomal drugs. This is the first report of in vitro and in vivo antitrypanosomal activities of either KS-505a or alazopeptin.
\end{abstract}

Keywords screening, in vitro, in vivo, antitrypanosomal antibiotics, Trypanosomal brucei brucei, T. b. rhodesiense, HAT

K. Otoguro (Corresponding author), A. Ishiyama, M. Namatame, A. Nishihara, T. Furusawa: Research Center for Tropical Diseases, Center for Basic Research, Kitasato University, 5-9-1 Shirokane, Minato-ku, Tokyo 108-8641, Japan, E-mail: otoguro@lisci.kitasato-u.ac.jp

S. Ōmura, H. Yamada, Y. Takahashi, K. Shiomi, R. Masuma: Kitasato Institute for Life Sciences and Graduate School of Infectious Control Sciences, Kitasato University, 5-9-1 Shirokane, Minato-ku, Tokyo 108-8641, Japan

M. Ichimura: BioFrontier Laboratories, Kyowa Hakko Kogyo Co., Ltd., 3-6-6 Asahi-machi, Machida-shi, Tokyo 194-8533, Japan

\section{Introduction}

Transmitted via the bite of tsetse flies (Glossina spp.) two sub-species of trypanosomes, Trypanosoma brucei rhodesiense and T. b. gambiense, infect humans and one sub-species, T. b. brucei, infects cattle. Human African Trypanosomiasis (HAT), also known as Sleeping Sickness, is recognized as one of African's most neglected diseases and is a major cause of mortality and morbidity in subSahara Africa. The cattle disease, nagana, devastates livestock production and causes massive economic losses. Accurate statistics for HAT are difficult to obtain. The World Health Organization (WHO) estimated that, in 2000, some 300,000 Africans were affected by the disease, a figure far in excess of the 27,000 cases reportedly diagnosed and treated that year. With increased surveillance activities in the last 7 years, recent estimates indicate there are 70,000 cases of HAT annually, causing 25,000 deaths $[1,2]$.

Currently, only four drugs (pentamidine, suramin, melarsoprol and eflornithine) are registered for the treatment of HAT. Pentamidine and suramin are used in the early stage of $T$. $b$. gambiense and $T . \quad b$. rhodesiense infections. Melarsoprol is used in the late-stage of both forms of the disease, while eflornithine is only used in the late-stage of $T$. $b$. gambiense infections. These drugs used to treat HAT are unsatisfactory, since they cannot be given orally and are hampered by severe toxicity. For example, melarsoprol is so poisonous that $5.0 \sim 10 \%$ of patients die because of toxic side effects [3]. Furthermore, drug resistance in trypanosomes is increasing and treatment failures are becoming more common [4, 5]. Therefore, there is an urgent need for new antitrypanosomal drugs that 
have novel structures and mechanisms of action, and which are both more effective and safer for chemotherapeutic control of HAT.

During our screening program to discover new antitrypanosomal antibiotics, we have been evaluating isolates from soil microorganisms and compounds from the antibiotic libraries of the Kitasato Institute for Life Sciences and BioFrontier Laboratories of Kyowa Hakko Kogyo Co., Ltd. We have previously reported on 10 microbial metabolites that exhibit selective and potent antitrypanosomal activities [6]. We have now discovered two compounds, KS-505a and alazopeptin, which show moderate antitrypanosomal characteristics. We report here in vitro antitrypanosomal activities, cytotoxicities and in vivo antitrypanosomal activities of KS-505a and alazopeptin, in comparison with commonly-used antitrypanosomal drugs.

\section{Materials and Methods}

\section{Parasites and History}

The history of $T$. b. brucei strain GUTat 3.1 and $T$. $b$. rhodesiense strain STIB900 were described previously [6]. T. b. brucei strain $\mathrm{S} 427$, a clone derived from an isolate from a Ugandan sheep [7], was donated by Prof. T. Kinoshita (Research Institute for Microbial Diseases, Osaka University, Japan). The original clone was kindly provided by Dr. G. A. M. Cross, Rockefeller University, USA.

\section{Chemicals}

Test antibiotics were obtained from the antibiotic libraries of the Kitasato Institute for Life Sciences and BioFrontier Laboratories of Kyowa Hakko Kogyo Co., Ltd. Suramin and eflornithine were provided by Prof. R. Brun (Swiss Tropical Institute, Switzerland). Pentamidine isothionate salt was obtained from Sigma-Aldrich Inc. (St. Louis, MO. USA). Iscove's Modified Dulbecco's Medium (IMDM, with L-glutamine and HEPES, without $\mathrm{NaHCO}_{3}$ ), Minimum Essential Medium (MEM) with Earle's salts, MEM nonessential amino acids solution and Penicillin-Streptomycin solution were obtained from Gibco Laboratories Life Technologies (Grand Island, N. Y. USA). Fetal Bovine Serum (FBS) was obtained from Sigma-Aldrich Inc. (St. Louis, MO. USA) and horse Serum (HS) was obtained from Gibco Laboratories Life Technologies (Grand Island, N. Y. USA). Alamar Blue reagent was obtained from Sigma-Aldrich Inc. (St. Louis, MO. USA). Other chemicals were commercially available and all of analytical grade.

\section{Animals}

Female CD1 mice (ICR), 20 25 g, were obtained from Charles River Japan, Inc. Animals were placed in groups of four per cage, kept in a room under negative pressure with flow of $0.1 \sim 0.2 \mathrm{~m} / \mathrm{second}$. The animal room was held at a temperature of $25 \pm 2{ }^{\circ} \mathrm{C}$ and $60 \pm 10 \%$ relative humidity. Animals are maintained on a diet of CE-2 (Clea, Japan Inc.) and water ad libitum.

\section{In Vitro Antitrypanosomal Assay against T. brucei Species}

In vitro antitrypanosomal activities for $T$. b. brucei strain GUTat 3.1 and T. b. rhodesiense strain STIB900 were described previously [6]. In brief, T. b. brucei strain GUTat 3.1 was cultured in IMDM with various supplements and $10 \%$ heat-inactivated $\mathrm{FBS}$ at $37^{\circ} \mathrm{C}$, under $5.0 \% \mathrm{CO}_{2} / 95 \%$ air. T. b. rhodesiense strain STIB900 was cultured in MEM with Baltz supplements containing 15\% heat-inactivated $\mathrm{HS}$ at $37^{\circ} \mathrm{C}$, under $5.0 \% \quad \mathrm{CO}_{2} / 95 \%$ air, according to the method of Baltz et al. [8]. Ninety five $\mu$ l of the trypanosomes suspension $\left(2.0 \sim 2.5 \times 10^{4}\right.$ trypanosomes $/ \mathrm{ml}$ for strain GUTat 3.1 or $2.0 \sim 3.0 \times 10^{4}$ trypanosomes $/ \mathrm{ml}$ for strain STIB 900) was seeded in a 96-well microplate, and $5.0 \mu \mathrm{l}$ of a test compound solution (dissolved in $5.0 \%$ dimethylsulfoxide) was added followed by incubation for 72 hours (long incubation-low inoculation test: LILIT). Ten $\mu \mathrm{l}$ of the fluorescent dye Alamar Blue was added to each well. After incubation for $3 \sim 6$ hours, the resulting solution was read at $528 / 20 \mathrm{~nm}$ excitation wavelength and $590 / 35 \mathrm{~nm}$ emission wavelength by a FLx800 fluorescent plate reader (Bio-Tek Instrument, Inc. Vermont, USA). Data were transferred into a spreadsheet program (Excel). The $\mathrm{IC}_{50}$ values were determined using a fluorescent plate reader software (KC-4, Bio-Tek). Successive subcultures were done in 24-well tissue culture plates under the same conditions.

\section{Cytotoxicity Tests on Human Diploid Cell Line (MRC-5 Cells)}

Cytotoxicity assay against MRC-5 cells was carried out as previously described [9].

\section{In Vivo Antitrypanosomal Assay against T. brucei Species}

T. brucei from in vitro culture were well adapted to repeated infection and passage in ICR mice. Infected blood (about $10^{8 \sim 9}$ trypanosomes $/ \mathrm{ml}$ ) was collected with a heparinized syringe from mouse heart under $\mathrm{CO}_{2}$ anesthesia. Collected blood was mixed gently with cryostabilant solution (1:1) consisting of phosphatebuffered saline containing 10\% glucose (PSG) and 10\% 
glycerol [10]. The cryostabilate was transferred to cryocapillary tubes and stored in liquid nitrogen before use.

\section{T. b. brucei S427 Acute Mouse Model}

Groups of four female ICR mice were infected intraperitoneally (i.p.) on day $0(\mathrm{~d} 0)$ with $10^{4}$ bloodstream forms of T. b. brucei strain S427, prepared in cryostabilate dilution with PSG. Infected mice were treated i.p. with each test compound (10\% DMSO-Tween 80/EtOH mixture ( $7: 3$ ) aqueous solution) on four consecutive days (d1 to d4). A control group remained untreated. Parasitaemia levels of the mice were checked by microscopic examination of tail blood on $\mathrm{d} 4$ after final treatment and thereafter twice a week until $\mathrm{d} 30$. The date of death of each mouse was recorded to calculate the mean survival day (MSD). Surviving and aparasitaemic mice at d30 were considered cured.

\section{T. b. rhodesiense STIB900 Acute Mouse Model}

Groups of four female ICR mice were infected i.p. on d0 with $3 \times 10^{4}$ bloodstream forms of $T$. $b$. rhodesiense strain STIB900 prepared from cryostabilate dilution with PSG. Infected mice were treated i.p. with each test compound (10\% DMSO-Tween 80/EtOH mixture (7:3) aqueous solution) on four consecutive days ( $\mathrm{d} 3$ to $\mathrm{d} 6$ ). A control group remained untreated. Parasitaemia levels of the mice were checked by microscopic examination of tail blood on d6 after final treatment and thereafter twice a week until d60. The date of death of each mouse was recorded to calculate the MSD. Surviving and aparasitaemic mice at d60 were considered cured.

\section{Results}

\section{In Vitro Antitrypanosomal Activities and Cytotoxicities of KS-505a and Alazopeptin}

In vitro antitrypanosomal activity of $\mathrm{KS}-505 \mathrm{a}$ and alazopeptin were evaluated on both $T$. b. brucei strain GUTat 3.1 and T. b. rhodesiense strain STIB900. The $\mathrm{IC}_{50}$ values of KS-505a, alazopeptin and three reference drugs (pentamidine, suramin and eflornithine) are listed in Table 1. KS-505a and alazopeptin possessed antitrypanosomal activities against strain GUTat 3.1, with the $\mathrm{IC}_{50}$ values of 1.03 and $0.51 \mu \mathrm{g} / \mathrm{ml}$, respectively. These antibiotics also possessed antitrypanosomal activities against strain STIB900, with $\mathrm{IC}_{50}$ values of 1.66 and $1.21 \mu \mathrm{g} / \mathrm{ml}$, respectively. The antitrypanosomal activities of these antibiotics for strains GUTat 3.1 and STIB900 were lower, by $319 \sim 1,107$-fold than those of pentamidine for the same strains, whereas, antitrypanosomal activities of these antibiotics for strains GUTat 3.1 and STIB900 were the same or similar to those of suramin and eflornithine for strain GUTat 3.1, and to that of eflornithine for strain STIB900 (Table 1).

We subsequently investigated the cytotoxicities of KS505a and alazopeptin against MRC-5 cells, with $\mathrm{IC}_{50}$ values also listed in Table 1. KS-505a and alazopeptin showed lower cytotoxicities with $\mathrm{IC}_{50}$ values of $>27.33$ and $>9.1 \mu \mathrm{g} / \mathrm{ml}$, respectively, and do not seem to be cytotoxic.

To compare the antitrypanosomal activities and cytotoxicities, we introduced the selectivity index (SI) listed in Table 1. KS-505a and alazopeptin showed moderate and lower SI, with ratios ranging between $>17.8 \sim>26.5$ and $>7.5 \sim>16.5$ for MCR-5 cells/strain GUTat 3.1 and MCR-5 cells/strain STIB900, respectively. These SI were lower than the commonly-used antitrypanosomal drugs, pentamidine, suramin and

Table 1 In vitro antitrypanosomal activity and cytotoxicity of KS-505a, alazopeptin and drugs used to treat Human African Trypanosomiasis

\begin{tabular}{|c|c|c|c|c|c|}
\hline \multirow{3}{*}{ Compound } & \multicolumn{3}{|c|}{$\mathrm{IC}_{50}(\mu \mathrm{g} / \mathrm{ml})$} & & \\
\hline & \multicolumn{2}{|c|}{ Antitrypanosomal activity } & \multirow{2}{*}{$\begin{array}{c}\text { Cytotoxicity } \\
\text { MRC-5 }\end{array}$} & \multicolumn{2}{|c|}{ Selectivity index (SI) } \\
\hline & GUTat 3.1 & STIB900 & & M/T. b. b. & M/T. b. r. \\
\hline KS-505a & 1.03 & 1.66 & $>27.33$ & $>26.5$ & $>16.5$ \\
\hline Alazopeptin & 0.51 & 1.21 & $>9.10$ & $>17.8$ & $>7.5$ \\
\hline Pentamidine & 0.0016 & 0.0015 & 5.71 & 3,569 & 3,807 \\
\hline Suramin & 1.58 & 0.052 & $>100$ & $>63$ & $>1,923$ \\
\hline Eflornithine & 2.27 & 1.04 & $>100$ & $>44$ & $>96$ \\
\hline
\end{tabular}


eflornithine.

\section{In Vivo Antitrypanosomal Activities of KS-505a and Alazopeptin}

KS-505a, alazopeptin and pentamidine were evaluated using the T. $b$. brucei $\mathrm{S} 427$ acute mouse model and T. $b$. rhodesiense STIB900 acute mouse model. Their cure rates and MSD are listed in Table 2. With the T. b. brucei strain S427, KS-505a cured all mice infected at a dose of $30 \mathrm{mg} / \mathrm{kg}$. In the case of a lower dose $(10 \mathrm{mg} / \mathrm{kg})$, cure was not achieved, but MSD was extended (13.0 days) which represented a 3-fold increase over control MSD (4.4 days). Conversely, alazopeptin (at a dose of $50 \mathrm{mg} / \mathrm{kg}$ ) did not achieve cure, but it also extended MSD (10.3 days), a 1.8fold increase over control MSD (5.8 days). Pentamidine, at a dose of $1.0 \mathrm{mg} / \mathrm{kg}$, cured all infected mice in this model. A lower dose $(0.2 \mathrm{mg} / \mathrm{kg})$ achieved only $25 \%$ cure but also showed an extended MSD ( $>17.3$ days) which was $>2.9$ fold longer than that of control MSD (6.0 days). Ten $\mathrm{mg} / \mathrm{kg}$ dosage of KS-505a exhibited similar antitrypanosomal activity to that of pentamidine $(0.2 \mathrm{mg} / \mathrm{kg})$ in the $T . b$. brucei S427 acute mouse model.

KS-505a was also tested with the $T$. b. rhodesiense STIB900 acute mouse model. In this model, KS-505a at a dose of $42 \mathrm{mg} / \mathrm{kg}$ achieved only $25 \%$ cure but did show an extended MSD (>28.5 days), which was $>3.6$-fold longer than that of control MSD (8.0 days) (Table 2). In the case of pentamidine, at a dose of $20 \mathrm{mg} / \mathrm{kg}$, the drug achieved $50 \%$ cure and exhibited an extended MSD ( $>49.3$ days), which was $>5.8$-fold longer than that of control MSD $(8.5$ days) in this model compared with the T. b. brucei $\mathrm{S} 427$ acute mouse (Table 2).

The KS-505a data suggest that it is possibly a new candidate compound for discovering new antitrypanosomal drugs with more potent activity.

\section{Discussion}

We discovered the antitrypanosomal activity of two microbial metabolites and evaluated this in vitro and in vivo using T. b. brucei strain GUTat 3.1 and T. b. rhodesiense strain STIB900. In the STIB900 acute mouse model, Baliani et al., suggested that parasites appear to leave the vasculature system early so that drugs must work in an extravascular fashion to effect radical cure. Therefore, this in vivo test represents a good model for evaluating likely outcomes of drugs in late-stage models [11]. Our results also indicated that the STIB900 acute mouse model was more stringent than the S427 acute mouse model in the case of pentamidine.

KS-505a (Fig. 1) is a tetraterpenoid antibiotic, and is reported to possess biological activities, including inhibition of brain $\mathrm{Ca}^{2+} /$ calmodulin-dependent cyclic nucleotide phosphodiesterase (CaM-PDE) and neuritepromoting activity in NG108-15 neuroblastoma $\times$ glioma hybrid cells [12, 13], as well, when administered intraperitoneally it exhibits in vivo anti-amnesia activity in an electroconvulsive shock-induced amnesia model in the rat [14]. In contrast to the mammalian PDEs, the function of PDEs in parasitic protozoa remains mostly unknown. With regard to Trypanosoma brucei species, Zoraghi et al. [15], Rascón et al. [16], and Zoraghi et al. [17] recently reported that T. brucei PDEs (TbPDE2 family: TbPDE2A, TbPDE2B and TbPDE2C) were found to be essential for bloodstream form trypanosome proliferation. Furthermore, Oberholzer et al., reported that TbPDE2B and TbPDE2C, as flagellar enzymes, were found to be essential for parasite virulence [18]. They also suggested that the TbPDE2 family might be considered as new molecular targets for chemotherapeutic antitrypanosomal drugs [15 18].

Table 2 In vivo antitrypanosomal activity of KS-505a, alazopeptin and pentamidine in two mouse models

\begin{tabular}{|c|c|c|c|c|c|}
\hline Compound & $\begin{array}{l}\text { Dosage } \\
(\mathrm{mg} / \mathrm{kg})\end{array}$ & Route & $\begin{array}{l}\text { No. of mice cured/ } \\
\text { No. of mice infected }\end{array}$ & MSD & $\begin{array}{l}\text { Control } \\
\text { MSD }\end{array}$ \\
\hline \multicolumn{6}{|c|}{ T. b. brucei S427 mouse model } \\
\hline KS-505a & $30 \times 4$ & i.p. & $4 / 4$ & $>30$ & 4.4 \\
\hline KS-505a & $10 \times 4$ & i.p. & $0 / 4$ & 13.0 & 4.4 \\
\hline Alazopeptin & $50 \times 4$ & i.p. & $0 / 4$ & 10.3 & 5.8 \\
\hline Pentamidine & $1 \times 4$ & i.p. & $4 / 4$ & $>30$ & 4.4 \\
\hline Pentamidine & $0.2 \times 4$ & i.p. & $1 / 4$ & $>17.3$ & 6.0 \\
\hline \multicolumn{6}{|c|}{ T. b. rhodesiense STIB 900 mouse model } \\
\hline KS-505a & $42 \times 4$ & i.p. & $1 / 4$ & $>28.5$ & 8.0 \\
\hline Pentamidine & $20 \times 4$ & i.p. & $2 / 4$ & $>49.3$ & 8.5 \\
\hline Pentamidine & $10 \times 4$ & i.p. & $1 / 4$ & $>45.8$ & 8.5 \\
\hline
\end{tabular}




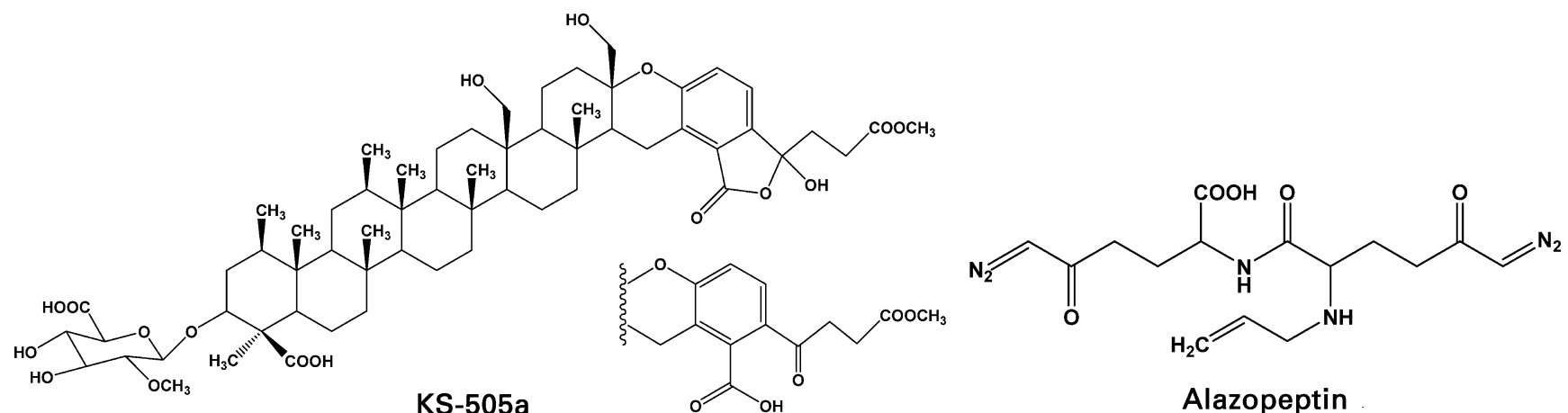

Fig. 1 Structures of KS-505a and alazopeptin.

Therefore, it may be useful to study T. brucei PDEs using $\mathrm{KS}-505 \mathrm{a}$ as an inhibitor of mammalian CaM-PDE. The relationship between mammalian PDEs and TbPDEs remains of significant interest.

Alazopeptin is an azaamino acid antibiotic [19], and is reported to have antitumor, antibacterial, and glutamine and purine antagonist activity [20]. Alazopeptin contains two moles of DON and alanine in the structure (Fig. 1). Recently, Hofer et al. reported that DON exhibited in vitro and moderate in vivo antitrypanosomal activities [21]. Its in vivo antitrypanosomal activity was static (not curative), furthermore, the mode of action of DON was via inhibition of CTP synthetase of T. brucei [21]. Alazopeptin may inhibit Trypanosma species in the same manners as DON.

The above results reveal that KS-505a and alazopeptin are promising lead compounds for new types of antitrypanosomal drugs. Further investigations of the antitrypanosomal potential of KS-505a and alazopeptin are in progress.

Acknowledgements This work was supported, in part, by funds from the Drugs for Neglected Diseases initiative (DND $i$ ), and a grant for All Kitasato Project Study (AKPS). We are grateful to Dr. Y. Yabu, Nagoya City University, Prof. R. Brun and Dr. M. Kaizer, Swiss Tropical Institute, Prof. T. Kinoshita, Research Institute for Microbial Diseases, Osaka University, and Prof. S. Croft, Dr. Eric Chatelain, Dr. J.-R. Ioset, Dr. C. Brünger and Miss F. Hirabayashi, DND $i$, for valuable discussions. We also thank Miss H. Sekiguchi (The Kitasato Institute) for her technical assistance throughout this work.

\section{References}

1. http://www.dndi.org/

2. WHO. Human African trypanosomiasis (sleeping sickness): epidemiological update. Wkly Epidemiol Rep 81: 71-80
(2006)

3. Fairlamb AH. Chemotherapy of human African trypanosomiasis: current and future prospects. Trends Parasitol 19: 488-494 (2003)

4. Matovu E, Seebeck T, Enyaru JCK, Kaminsky R. Drug resistance in Trypanosoma brucei spp., the causative agents of sleeping sickness in man and nagana in cattle. Microbes Infect 3: 763-770 (2001)

5. Brun R, Schumacher R, Schmid C, Kunz C, Burri C. The phenomenon of treatment failures in Human African Trypanosomiasis. Trop Med Int Health 6: 906-914 (2001)

6. Otoguro K, Ishiyama A, Namatame M, Nishihara A, Furusawa T, Masuma R, Shiomi K, Takahashi Y, Yamada H, Ömura S. Selective and potent in vitro antitrypanosomal activities of ten microbial metabolites. J Antibiot 61: 372-378 (2008)

7. Cunningham MP, Vickerman K. Antigenic analysis in Trypanosoma brucei group using an agglutination reaction. Trans R Soc Trop Med Hyg 56: 48-59 (1962)

8. Baltz T, Baltz D, Giroud Ch, Crockett J. Cultivation in a semi-defined medium of animal infective forms of Trypanosoma brucei, T. equiperdum, T. evansi, T. rhodesiense and T. gambiense. EMBO J 4: 1273-1277 (1985)

9. Otoguro K, Kohana A, Manabe C, Ishiyama A, Ui H, Shiomi K, Yamada H, Ōmura S. Potent antimalarial activities of polyether antibiotic, X-206. J Antibiot 54: 658-663 (2001)

10. Lanham S, Godfrey DG. Isolation of salivarian trypanosomes from man and other mammals using DEAECellulose. Exp Parasitol 28: 521-534 (1970)

11. Baliani A, Bueno GJ, Stewart ML, Yardley V, Brun R, Barrett MP, Gilbert IH. Design and synthesis of a series of melamine-based nitroheterocycles with activity against Trypanosomatid parasites. J Med Chem 48: 5570-5579 (2005)

12. Nakanishi S, Osawa K, Saito Y, Kawamoto I, Kuroda K, Kase H. KS-505a, a novel inhibitor of bovine brain $\mathrm{Ca}^{2+}$ and calmodulin-dependent cyclic-nucleotide phosphodiesterase from Streptomyces argenteolus. J Antibiot 45: 341-347 
(1992)

13. Nagashima K, Toki S, Nakanishi S, Kase H, Matsuda Y. Neurite formation in NG108-15 neuroblastoma $\times$ glioma hybrid cells by KS-505a, a potent inhibitor of brain cyclicnucleotide phosphodiesterase. J Antibiot 46: 1481-1483 (1993)

14. Ichimura M, Eiki R, Osawa K, Nakanishi S, Kase H. KS$505 \mathrm{a}$, an isoform-selective inhibitor of calmodulindependent cyclic-nucleotide phosphodiesterase. Biochem J 316: 311-316 (1996)

15. Zoraghi R, Kunz S, Gong K, Seebeck T. Characterization of TbPDE2A, a novel cyclic nucleotide-specific phosphodiesterase from the Protozoan parasite Trypanosoma brucei. J Biol Chem 276: 11559-11566 (2001)

16. Rascón A, Scoderling SH, Schaefer JB, Beavo JA. Cloning and characterization of a cAMP-specific phosphodiesterase (TbPDE2B) from Trypanosoma brucei. Proc Natl Acad Sci USA 99: 4714-4719 (2002)

17. Zoraghi R, Kunz S, Gong K, Seebeck T. The cAMP-specific phosphodiesterase TbPDE2C is an essential enzyme in bloodstream from Trypanosoma brucei. Proc Natl Acad Sci USA 99: 4343-4348 (2002)

18. Oberholzer M, Marti G, Beresic M, Kunz S, Hemphill A, Seebeck T. The Trypanosoma brucei cAMP phosphodiesterases TbrPDEB1 and TbrPDEB2: flagellar enzymes that are essential for parasite virulence. FASEB J 21: 720-731 (2007)

19. Petterson EL, Johnson BL, DeVoe SE, Bohonos N. Structure of the antitumor antibiotic alazopeptin. Antimicrob Agents Chemother 5: 115-118 (1965)

20. DeVoe SE, Rigler NE, Shay AJ, Martin JH, Boyd TC, Backus EJ, Mowat JH, Bohonos N. Alazopeptin; production, isolation, and chemical characteristics. Antibiotics Annual 1956-1957: 730-735

21. Hofer A, Steverding D, Chabes A, Brun R, Thelander L. Trypanosoma brucei CTP synthetase: A target for the treatment of African sleeping sickness. Proc Natl Acad Sci USA 98: 6412-6416 (2001) 